Title:

\title{
Correlating Patient Anxiety with Urinary Symptoms before and after Holmium Laser Enucleation of the Prostate
}

Authors: Charles U. Nottingham, Deepak K. Agarwal, Crystal Valadon, Tim Large, Marcelino E. Rivera, Amy E. Krambeck

Charles U. Nottingham, MD

cunottingham@gmail.com

Department of Urology, Indiana University School of Medicine

1801 Senate Blvd. Suite 220

Indianapolis, IN 46202

Phone: 1-317-962-3700

Fax: 1-317-962-2893

Deepak K. Agarwal, MD

dkagarwa@iu.edu

Department of Urology, Indiana University School of Medicine 1801 Senate Blvd. Suite 220

Indianapolis, IN 46202

Phone: 1-317-962-3700

Fax: 1-317-962-2893

Crystal Valadon

cvaladon@iu.edu

Department of Urology, Indiana University School of Medicine 
1801 Senate Blvd. Suite 220

Indianapolis, IN 46202

Phone: 1-317-962-3700

Fax: 1-317-962-2893

Tim Large, MD

tlarge@iuhealth.org

Department of Urology, Indiana University School of Medicine 1801 Senate Blvd. Suite 220

Indianapolis, IN 46202

Phone: 1-317-962-3700

Fax: 1-317-962-2893

Marcelino E. Rivera, MD

mrivera7@iuhealth.org

Department of Urology, Indiana University School of Medicine 1801 Senate Blvd. Suite 220

Indianapolis, IN 46202

Phone: 1-317-962-3700

Fax: 1-317-962-2893

Amy E. Krambeck, MD

akrambeck@iuhealth.org

Department of Urology, Indiana University School of Medicine 
1801 Senate Blvd. Suite 220

Indianapolis, IN 46202

总

Phone: 1-317-962-3700

Fax: 1-317-962-2893

Please address all correspondence to:

Charles U. Nottingham, MD

cunottingham@gmail.com

Department of Urology, Indiana University School of Medicine

1801 Senate Blvd. Suite 220

Indianapolis, IN 46202

Phone: 1-317-962-3700

Fax: 1-317-962-2893

Keywords: anxiety; lower urinary tract symptoms; benign prostate hyperplasia; holmium laser enucleation of the prostate 


\begin{abstract}
Introduction and Objective:

Preoperative anxiety has been shown to correlate with certain urinary symptoms in patients with certain types of voiding dysfunction, particularly overactive bladder. The purpose of this study was to evaluate if patient-reported anxiety correlates with pathologic characteristics, voiding symptoms, and objective metrics of bladder emptying in male patients undergoing holmium laser enucleation of the prostate (HoLEP).
\end{abstract}

\title{
Patients and Methods:
}

We began collecting patient-reported anxiety scores (GAD-7) in addition to patientreported scores of lower urinary tract symptoms (LUTS) and erectile dysfunction (ED) starting in November 2018 for men with LUTS due to benign prostate hyperplasia. We retrospectively evaluated the relationship between preoperative GAD-7 score with preoperative age, BMI, preoperative urine flow metrics, preoperative post-void residual (PVR), and preoperative symptom scores for LUTS (AUA, BII, and MISI) and ED (SHIM and MSHQ-EjD). We then compared preoperative GAD-7 score to enucleated prostate weight, post-operative GAD-7 scores, post-operative urine flow metrics, post-operative PVR, and postoperative symptom scores for LUTS and ED.

Results:

We included 139 patients for analysis. The preoperative GAD-7 score positively correlated with preoperative BII scores $\left(r_{s}=0.202, p=0.019\right)$, MISI total $\left(r_{s}=0.260, p=0.002\right)$ and MISI bother $\left(r_{s}=0.345, p<0.001\right)$ scores. Preoperative GAD-7 negatively correlated with preoperative MSHQ-EJD $\left(r_{s}=-0.191, p=0.031\right)$. Following HoLEP, all four of these correlations disappear. The GAD-7 score did not correlate with age, BMI, preoperative urine flow metrics, preoperative PVR, or other preoperative LUTS and ED symptom scores. Additionally, preoperative GAD-7 score showed no correlation with enucleated prostate weight, postoperative urine flow metrics, postoperative PVR, and postoperative LUTS and ED symptom scores. 
Conclusions:

Higher preoperative anxiety correlated with more severe pre-operative urinary LUTS, particularly incontinence symptoms, in patients undergoing HoLEP. Following HoLEP, these symptoms improve regardless of patient anxiety level. 
Introduction

Anxiety is a common condition in the modern era, with up to $32 \%$ of adolescents and adults carrying some form of anxiety disorder ${ }^{1}$. Patients with anxiety and other mental health disorders also seek urologic care for conditions such as lower urinary tract symptoms (LUTS) and erectile dysfunction (ED), and these two seemingly unrelated body systems may actually contribute to each other's disease processes ${ }^{2-4}$. In females, several studies have suggested that both depression and anxiety may specifically correlate with the diagnoses of overactive bladder $(O A B)$ and urinary incontinence ${ }^{3,5-7}$. Male patients with depression and anxiety may similarly experience urinary storage symptoms more commonly than men without these diagnoses ${ }^{8,5}$. However, correlations to obstructive LUTS show variability within the literature $\mathrm{e}^{8,9,10(\mathrm{p}), 11,12}$.

The correlations between LUTS in men and mental health disorders do not immediately reveal whether one of these two disease categories may play a causative role in the etiology of the other ${ }^{13,14}$. Additionally, few studies exist demonstrating that treatment of one condition will simultaneously treat the other. At our institution, we have recognized that patient anxiety may play a role in how to optimally treat the offending symptoms and in health-related quality of life (HRQoL) $)^{11,15}$. As such, we have recently included routine evaluation of anxiety using the validated generalized anxiety disorder 7item (GAD-7) ${ }^{16}$ score in male patients seeking treatment for LUTS due to benign prostate hyperplasia (BPH). In the present study, we correlate patient anxiety with LUTS both before and after surgical treatment with holmium laser enucleation of the prostate (HoLEP).

\section{Materials and Methods:}

This study was approved by the institutional review board at Indiana University (\# 1902628723). Clinical data on patients at our institution undergoing HoLEP is prospectively collected in a REDCap database ${ }^{17}$. As part of our pre-operative clinical visit, we perform a detailed history and physical examination. In addition, we have patients complete a packet of validated symptom assessment tools regarding their voiding and sexual function. Specifically, we include the international prostate symptom score (IPSS) including the IPSS 
quality of life (IPSS QoL $)^{18}$, the BPH impact index $(\mathrm{BII})^{19}$, Michigan incontinence symptom index (MISI) total and bother scores ${ }^{20}$, sexual health inventory for men (SHIM) ${ }^{21}$, and the male sexual health questionnaire for ejaculatory dysfunction (MSHQ-EjD) ${ }^{22}$. Cumulatively, we refer to these symptom assessment tools as the symptom scores for LUTS and erectile dysfunction (ED). Beginning in November 2018, we incorporated the GAD-7 ${ }^{16}$ score into the packet of validated questionnaires. We also measure urine flow metrics (maximal and average urine flow) and post-void residual (PVR) bladder volume by ultrasound. Postoperatively, patients return to the office approximately 3 months from the date of surgery. At this time we obtain symptom scores for GAD-7, LUTS and ED, as well as urine flow metrics and a PVR.

We included patients who had completed all of the symptom scores for LUTS and ED at both the pre-operative and the post-operative office visits, as well as a pre-operative GAD-7 score. Patients who did not return to our office for post-operative visits because they live too far away to return but mailed in the completed LUTS and ED symptom scores were also included. We correlated pre-operative GAD-7 scores with patient age, BMI, enucleated prostate weight, pre-operative and post-operative symptom scores for LUTS and ED, as well as preoperative and postoperative urine flow metrics and PVR values. Patients with indwelling catheters and therefore could not provide urine flow metrics or PVR did not have these specific variables included in the analysis, but their other data was included for analysis. We performed a sub-analysis comparing pre-operative and postoperative individual symptom scores from the IPSS, as well as a sub-analysis correlating pre-operative GAD-7 scores with individual components of the IPSS.

Of note, we did not exclude patients due to a past or present diagnosis of anxiety. Furthermore, we did we exclude patients currently or previously taking anxiolytics. We assessed anxiety as a continuous variable based on the GAD-7 score, given that it is a validated assessment of anxiety levels in all patients and would be the most up to date assessment of patients with existing diagnoses of anxiety and patients with undiagnosed anxiety. 
A complete description of the HoLEP procedure at our institution has been previously described ${ }^{23}$. Briefly, we use a 120 watt holmium:YAG laser (Pulse; Lumenis, Inc.) with a 550 or $1000 \mu \mathrm{m}$ diameter laser fiber. We typically enucleate the median lobe first, and then perform a bottom-up approach.

All statistical analysis was performed using IBM SPSS software, version 25 (Armonk, NY). Mean and standard deviation values were calculated for patient age, BMI, and enucleated prostate weight. We compared mean preoperative and postoperative GAD-7 scores, symptom scores for LUTS and ED, urine flow metrics, and PVR using a two-tailed, paired Student's t-test. Spearman's correlation coefficient test was used to correlate preoperative GAD-7 scores with preoperative and postoperative symptom scores for LUTS and ED, as well as preoperative and postoperative urine flow metrics and PVR values. Significance was determined with a $p$-value less than 0.05 .

\section{Results}

We included a total of 139 patients for analysis. The respective means (standard deviations) for age, BMI, and enucleated prostate weight among the group were 69 years (9 years), $28.7 \mathrm{~kg} / \mathrm{m}^{2}\left(5.2 \mathrm{~kg} / \mathrm{m}^{2}\right.$ ), and 61 grams (50 grams). Forty-four (31.7\%) patients had a preoperative GAD-7 score of 0 , and 94 (67.6\%) had scores in the minimal anxiety range of $0-4^{16}$. Twenty-five (18.0\%) patients had a GAD-7 score of 5-9 corresponding to mild anxiety, ten (7.2\%) had scores in the moderate anxiety range of $10-14$, and ten (7.2\%) had scores of 15 or higher corresponding to severe anxiety.

Table 1 compares mean preoperative and postoperative questionnaire scores, urine flow metrics, and PVR. The mean pre-operative GAD-7 score was 4, which postoperatively declined to $3(p<0.001)$. The preoperative mean IPSS of 20 fell into the severely symptomatic range, but postoperatively this declined to 9 (moderately symptomatic range). Preoperative IPSS QoL scores also declined from a mean of 4 (mostly dissatisfied) to a mean of 2 (mostly satisfied) postoperatively. Bll scores decreased from a mean of 7 preoperatively to 3 postoperatively. MISI total scores increased from 7 to 9 postoperatively, while MISI bother scores remained stable at 2. SHIM scores remained stable, while MSHQ-EJD scores decreased fro 8 to 6 postoperatively. Urine flow metrics 
showed significantly increased maximal flow rates postoperatively, although average flow rates were not significantly different. PVR did significantly decrease from postoperatively. Twenty-three patients (16.5\%) were in urinary retention requiring an indwelling catheter or intermittent catheterization prior to surgery. Of these men in retention, their mean postoperative peak urine flow rate was 11.6 (SD 6.7) $\mathrm{mL} / \mathrm{second}$, their mean postoperative average urine flow rate was 5.2 (SD 3.1) $\mathrm{mL} /$ second, and their mean post-void residual was 105 (SD 196) mL.

Table 2 contains correlations between preoperative GAD-7 scores patient demographics, preoperative and postoperative LUTS and ED scores, preoperative and postoperative urine flow metrics and PVR, and enucleated prostate weight using Spearman's correlation coefficient. The preoperative GAD-7 score positively correlated with preoperative BII scores $\left(r_{s}=0.202, p=0.019\right)$, MISI total $\left(r_{s}=0.260, p=0.002\right)$ and MISI bother $\left(r_{s}=0.345, p<0.001\right)$ scores, meaning a higher GAD-7 correlated with worse LUTS and worse incontinence. Preoperative GAD-7 negatively correlated with preoperative MSHQ-EJD $\left(r_{s}=-0.191, p=0.031\right)$, meaning the higher the GAD-7 the more severe the preoperative ejaculatory dysfunction. We observed no correlation with patient age, BMI, and enucleated prostate weight. Following HoLEP, these correlations disappeared. Additionally, we observed no correlation between preoperative GAD-7 and postoperative IPSS, IPSS QoL, SHIM, urine flow metrics, and PVR. Preoperative and postoperative GAD-7 scores had a significantly positive correlation $\left(r_{s}=0.571, p<0.001\right)$, suggesting no change in patient-reported anxiety before and after surgery.

A sub-analysis comparing mean pre-operative and post-operative individual symptom scores within the IPSS is presented in Supplementary Table 1. Mean symptom scores for all parameters were significantly higher preoperatively compared to postoperatively (all $p<0.001$ ). A sub-analysis correlating pre-operative GAD-7 to individual symptom scores within the IPSS, both pre-operatively and post-operatively, is presented in Supplementary Table 2. Pre-operative GAD-7 positively correlated with pre-operative urgency $\left(r_{s}=0.185, p<0.033\right)$, pre-operative straining $\left(r_{s}=0.173, p<0.048\right)$, and postoperative urgency $\left(r_{s}=0.201, p<0.045\right)$. 


\section{Discussion}

We retrospectively evaluated patients undergoing HoLEP for LUTS due to enlarged prostate, and explored correlations between patient anxiety and preoperative and postoperative LUTS and ED. We observed that prior to HoLEP, higher patient anxiety positively correlated with more severe voiding symptoms including incontinence, as well as with worse preoperative ejaculatory dysfunction. We then observed that these correlations disappear postoperatively and those patients with a higher GAD-7 score could obtain the same surgical results as patients with lower GAD-7 scores. In other words, preoperative anxiety may have resulted in worse voiding symptoms prior to surgery but did not affect the surgical outcome. Based on the dramatic improvement of LUTS following HoLEP in this series and others ${ }^{24}$, we can speculate that voiding symptoms would contribute less to a patient's anxiety. The anxiety captured by the GAD-7 score was preserved postoperatively and occurs independent of LUTS based on the strong and positive correlation between preoperative and postoperative GAD-7. The symptoms that appear to be most impacted by anxiety are urgency, straining, and incontinence, as well as the overall effect of BPH on health (as measured by the BII).

As surgical specialists, it is important to recognize that the urologic conditions for which our patients seek treatment may not exist in isolation from anxiety and other mental health disorders. As it pertains to the present study, the impact of LUTS on patient quality of life is especially important. Previously, a study from France assessed the change in both urinary symptoms and health-related quality of life (HRQoL), as measured with the EQ-5D score, as a response to medical treatment of LUTS $^{13}$. Over a mean of 5.2 years, patient quality of life outcomes were overall quite disappointing. Worse IPSS quality of life scores were reported by $42 \%$ of patients, and patients with moderate-to-severe LUTS had altered HRQoL scores in all five parameters of the EQ-5D. Similarly, Yang et $a^{25}$ found that patients with anxiety and BPH more commonly had no response of their IPSS QoL scores when treated medically for BPH. Taken together, these may suggest that medical treatment of LUTS due to BPH in patients with anxiety may poorly respond to medical therapies in terms of urinary quality of life. Looking more broadly at the impact of LUTS on various aspects of a patient's life, Lee et al ${ }^{15}$ examined correlations between HRQoL and 
patient-reported OAB and LUTS in a survey-based study of over 8,000 patients in China, Taiwan, and South Korea. Interestingly, patients with LUTS also reported lower workplace productivity, presumably due to the burdens associated with their disease. Our study did not find a correlation with the IPSS quality of life score, but did see a positive correlation with BII. Our patient population that is ready for surgical management typically has high preoperative IPSS and IPSS QoL scores for the entire population, which may explain why there was no correlation with preoperative GAD-7. Similar to these aforementioned studies of HRQoL, the Bll asks the patient to think about voiding in the context of his entire health. Our positive correlation between BII and GAD-7 scores appears to fit with the data from those other studies of HRQoL such that patients with higher anxiety may experience more concern over how their voiding symptoms affect their overall health.

As reviewed above, prior authors have observed that patient incontinence and $O A B$ symptoms correlate positively with patient anxiety in both female and male patients. The reason for this association remains unclear. Lai et $\mathrm{al}^{26}$ compared male and female patients with $O A B$ to a matched cohort of patients without $O A B$, and found that patients with $O A B$ reported more systemic (i.e. non-urological) symptoms. The authors suggested that perhaps there exist systemic factors contributing to both the LUTS and the non-urological symptoms. The correlation between LUTS and systemic factors has been explored by Martin et $\mathrm{al}^{8}$, who measured serum inflammatory marker levels in patients with LUTS and coexistent depression and/or anxiety, albeit with inconclusive results. Others have suggested that perhaps there is a psychogenic component ${ }^{27}$. In particular, patients with psychogenic urinary dysfunction may experience transient or situational urodynamic findings consistent with $\mathrm{OAB}$ or other symptoms ${ }^{28}$.

Of particular importance, patient-reported incontinence on the MISI scores preoperatively held an association with anxiety, but this association was lost following HoLEP. Meaning, patients with anxiety were not likely to experience more severe postoperative incontinence. A prior study by Felde et al $^{6}$ suggested that both depression and anxiety are independent risk factors for incontinence in a large Norwegian female cohort of over 16,000 patients. Interestingly, they observed the anxiety association with both stress and urge incontinence. While the etiology for female incontinence is likely not 
directly comparable to BPH in men, there may be some overlap in the incontinence pathways common to both sexes. One element of preoperative incontinence in our male cohort is the element of post-void dribbling, which anecdotally many of our patients categorize as incontinence and for which they will wear pads. While this type of incontinence may differ from stress or urge incontinence, it is still a bothersome symptom for patients causing them to seek treatment. The results of our present study would support that the character of incontinence preoperative and postoperatively may be different, and the experience of incontinence to the patient may be different depending on patient anxiety levels.

Contrary to our suspicions, prostate size did not correlate with anxiety. Given the positive correlation between LUTS and anxiety, we suspected that patients with higher levels of anxiety may experience bothersome LUTS earlier in the disease process of benign prostate growth, i.e. while the prostate is still relatively small. As our data demonstrates, this correlation did not exist. The lack of association suggests that the LUTS must develop independent of anxiety. While the severity of LUTS as measured by validated (and numerical) symptom scores may be more pronounced in men with anxiety, the biologic phenomenon of benign prostate hyperplasia must occur for men to experience obstructive LUTS related to this condition. This would suggest that anxiety independently does not play a causative role in LUTS, but rather correlates with a more severe patient experience of LUTS. This may be important when assessing patients both at the initial encounter and in follow-up, as these patients may benefit from a more frequent or aggressive management strategy to control their symptoms.

Our study does have certain limitations. First, it is the retrospective nature of our analysis; however, all data is prospectively maintained in a dedicated database that is updated weekly. Second, our assessment of patient anxiety relies upon patients accurately and truthfully completing these validated questionnaires. It is possible that patients may underreport anxiety if they are reluctant to complete the anxiety questionnaires accurately for any reason such mental health disease stigma or desire to focus solely on the urologic issue at hand. Third, we are a tertiary referral center for HoLEP and often see patients who are in a more advanced state of their LUTS who are ready for surgical 
intervention. Our cohort assessed patients ready for a surgical intervention, and we did not assess correlations of anxiety, LUTS, and ED at earlier stages in the disease process when a less-invasive intervention such as initiation of or change in medication would be offered. Similarly, we did not evaluate other surgical treatments for LUTS due to BPH. Additionally, this is a male cohort treated for BPH, which is one of many etiologies of LUTS. Consequently, we did not assess the response to other surgical interventions for different causes of LUTS such as those experienced in a female population. Fourth, since we are a tertiary referral center the outcomes may be biased towards patients who are experiencing a prolonged recovery after surgery. In general, patients that are pleased with their results are more likely to cancel their 3 month appointment while those that are still in the recovery process tend to keep the appointment. The follow up confounder most likely accounts for the higher MISI score at 3 month follow up. We tried to correct for this confounding factor by mailing the questionnaires to patients who did not keep their follow up appointments. Fifth, the GAD-7 score is a tool to independently assess anxiety, but not assess anxiety as it relates to voiding symptoms. This is demonstrated by the strong positive correlation seen between preoperative and postoperative GAD-7 scores in Table 2. An interesting future study could assess anxiety as it relates specifically to voiding symptoms, and assess if intervention for LUTS improves this disease-related anxiety. Despite these limitations this study demonstrates that surgical intervention can improve LUTs secondary to BPH even in patients with significant preoperative anxiety.

\section{Conclusions}

Higher preoperative anxiety positively correlated with more severe preoperative LUTS and ejaculatory dysfunction. Following HoLEP, these associations disappear in spite of anxiety levels remaining similar, many LUTS scores improving, and ejaculatory dysfunction being similar. Thus, patients with anxiety and LUTs will likely have worse preoperative symptoms compared to patients without anxiety, but they can expect to achieve similar surgical outcomes as patients without anxiety when treated with HoLEP.

\section{Acknowledgements}

None. 


\section{Reference:}

1. Essau CA, Lewinsohn PM, Lim JX, Ho M-HR, Rohde P. Incidence, recurrence and comorbidity of anxiety disorders in four major developmental stages. J Affect Disord. 2018;228:248-253. doi:10.1016/j.jad.2017.12.014

2. Breyer BN, Shindel AW, Erickson BA, Blaschko SD, Steers WD, Rosen RC. The association of depression, anxiety and nocturia: a systematic review. J Urol. 2013;190(3):953-957. doi:10.1016/j.juro.2013.03.126

3. Bradley CS, Nygaard IE, Hillis SL, Torner JC, Sadler AG. Longitudinal associations between mental health conditions and overactive bladder in women veterans. Am J Obstet Gynecol. 2017;217(4):430.e1-430.e8. doi:10.1016/j.ajog.2017.06.016

4. Nguyen HMT, Gabrielson AT, Hellstrom WJG. Erectile Dysfunction in Young Men-A Review of the Prevalence and Risk Factors. Sex Med Rev. 2017;5(4):508-520. doi:10.1016/j.sxmr.2017.05.004

5. Lai HH, Rawal A, Shen B, Vetter J. The Relationship Between Anxiety and Overactive Bladder or Urinary Incontinence Symptoms in the Clinical Population. Urology. 2016;98:5057. doi:10.1016/j.urology.2016.07.013

6. Felde G, Ebbesen MH, Hunskaar S. Anxiety and depression associated with urinary incontinence. A 10-year follow-up study from the Norwegian HUNT study (EPINCONT). Neurourol Urodyn. 2017;36(2):322-328. doi:10.1002/nau.22921

7. Mutungi S, Parrish J, Maunder R, et al. Is overactive bladder independently associated with anxiety? Int Urogynecology J. July 2019. doi:10.1007/s00192-019-04018-4

8. Martin S, Vincent A, Taylor AW, et al. Lower Urinary Tract Symptoms, Depression, Anxiety and Systemic Inflammatory Factors in Men: A Population-Based Cohort Study. PloS One. 2015;10(10):e0137903. doi:10.1371/journal.pone.0137903 
9. Koh JS, Ko HJ, Wang S-M, et al. The Relationship between Depression, Anxiety, Somatization, Personality and Symptoms of Lower Urinary Tract Symptoms Suggestive of Benign Prostatic Hyperplasia. Psychiatry Investig. 2015;12(2):268-273. doi:10.4306/pi.2015.12.2.268

10. Lung-Cheng Huang C, Ho C-H, Weng S-F, Hsu Y-W, Wang J-J, Wu M-P. The association of healthcare seeking behavior for anxiety and depression among patients with lower urinary tract symptoms: a nationwide population-based study. Psychiatry Res. 2015;226(1):247-251. doi:10.1016/j.psychres.2014.12.056

11. Choi EPH, Lam CLK, Chin WY. Mental Health Mediating the Relationship Between Symptom Severity and Health-Related Quality of Life in Patients with Lower Urinary Tract Symptoms. Low Urin Tract Symptoms. 2016;8(3):141-149. doi:10.1111/luts.12086

12. Huang $\mathrm{CL}-\mathrm{C}, \mathrm{Wu} \mathrm{M}-\mathrm{P}, \mathrm{Ho} \mathrm{C}-\mathrm{H}$, Wang J-J. The bidirectional relationship between anxiety, depression, and lower urinary track symptoms: A nationwide population-based cohort study. J Psychosom Res. 2017;100:77-82. doi:10.1016/j.jpsychores.2017.07.008

13. Fourcade R-O, Lacoin F, Rouprêt $\mathrm{M}$, et al. Outcomes and general health-related quality of life among patients medically treated in general daily practice for lower urinary tract symptoms due to benign prostatic hyperplasia. World J Urol. 2012;30(3):419-426. doi:10.1007/s00345-011-0756-2

14. Drossaerts J, Vrijens D, Leue C, Schilders I, Van Kerrebroeck P, van Koeveringe G. Screening for depression and anxiety in patients with storage or voiding dysfunction: $A$ retrospective cohort study predicting outcome of sacral neuromodulation. Neurourol Urodyn. 2016;35(8):1011-1016. doi:10.1002/nau.22871

15. Lee K-S, Yoo TK, Liao L, et al. Association of lower urinary tract symptoms and OAB severity with quality of life and mental health in China, Taiwan and South Korea: results from a cross-sectional, population-based study. BMC Urol. 2017;17(1):108. doi:10.1186/s12894-017-0294-3 
16. Spitzer RL, Kroenke K, Williams JBW, Löwe B. A brief measure for assessing generalized anxiety disorder: the GAD-7. Arch Intern Med. 2006;166(10):1092-1097. doi:10.1001/archinte.166.10.1092

17. Harris PA, Taylor R, Thielke R, Payne J, Gonzalez N, Conde JG. Research electronic data capture (REDCap)--a metadata-driven methodology and workflow process for providing translational research informatics support. J Biomed Inform. 2009;42(2):377-381. doi:10.1016/j.jbi.2008.08.010

18. Barry MJ, Fowler FJ, O'Leary MP, et al. The American Urological Association symptom index for benign prostatic hyperplasia. The Measurement Committee of the American Urological Association. J Urol. 1992;148(5):1549-1557; discussion 1564. doi:10.1016/s0022-5347(17)36966-5

19. Barry MJ, Fowler FJ, O'Leary MP, Bruskewitz RC, Holtgrewe HL, Mebust WK. Measuring disease-specific health status in men with benign prostatic hyperplasia. Measurement Committee of The American Urological Association. Med Care. 1995;33(4 Suppl):AS145-155.

20. Suskind AM, Dunn RL, Morgan DM, DeLancey JOL, McGuire EJ, Wei JT. The Michigan Incontinence Symptom Index (M-ISI): a clinical measure for type, severity, and bother related to urinary incontinence. Neurourol Urodyn. 2014;33(7):1128-1134. doi:10.1002/nau.22468

21. Rosen RC, Cappelleri JC, Smith MD, Lipsky J, Peña BM. Development and evaluation of an abridged, 5-item version of the International Index of Erectile Function (IIEF-5) as a diagnostic tool for erectile dysfunction. Int J Impot Res. 1999;11(6):319-326.

22. Rosen RC, Catania JA, Althof SE, et al. Development and validation of four-item version of Male Sexual Health Questionnaire to assess ejaculatory dysfunction. Urology. 2007;69(5):805-809. doi:10.1016/j.urology.2007.02.036

23. Rivera ME, Lingeman JE, Krambeck AE. Holmium Laser Enucleation of the Prostate. J Endourol. 2018;32(S1):S7-S9. doi:10.1089/end.2017.0710 
24. Rieken M, Bachmann A, Shariat SF. Long-term follow-up data more than 5 years after surgical management of benign prostate obstruction: who stands the test of time? Curr Opin Urol. 2016;26(1):22-27. doi:10.1097/MOU.0000000000000244

25. Yang YJ, Koh JS, Ko HJ, et al. The influence of depression, anxiety and somatization on the clinical symptoms and treatment response in patients with symptoms of lower urinary tract symptoms suggestive of benign prostatic hyperplasia. J Korean Med Sci. 2014;29(8):1145-1151. doi:10.3346/jkms.2014.29.8.1145

26. Lai HH, Vetter J, Jain S, Andriole GL. Systemic Nonurological Symptoms in Patients with Overactive Bladder. J Urol. 2016;196(2):467-472. doi:10.1016/j.juro.2016.02.2974 27. Sakakibara R, Ito T, Yamamoto T, et al. Depression, Anxiety and the Bladder. Low Urin Tract Symptoms. 2013;5(3):109-120. doi:10.1111/luts.12018

28. Sakakibara R, Uchiyama T, Awa $\mathrm{Y}$, et al. Psychogenic urinary dysfunction: a uroneurological assessment. Neurourol Urodyn. 2007;26(4):518-524. doi:10.1002/nau.20321 


\section{Abbreviations}

BII = Benign prostate hyperplasia impact index

$\mathrm{BPH}=$ Benign prostate hyperplasia

$E D=$ Erectile dysfunction

HoLEP $=$ Holmium laser enucleation of the prostate

HRQoL = Health related quality of life

GAD-7 = Generalized anxiety disorder 7-item

IPSS = International prostate symptom core

IPSS QoL = International prostate symptom score quality of life

LUTS = Lower urinary tract symptoms

MISI = Michigan incontinence symptom index

MSHQ-EjD = Male sexual health questionnaire for ejaculatory dysfunction

$\mathrm{OAB}=$ Overactive bladder

PVR = Post-void residual

SHIM = Sexual health inventory for men 
Table 1. Comparison of preoperative and postoperative GAD-7 score, LUTS scores, ED scores, urine flow metrics, and PVR.

\begin{tabular}{|l|l|l|l|}
\hline & Preoperative & Postoperative & p-value \\
\hline Mean (SD) GAD-7 & $\mathbf{4 ( 5 )}$ & $\mathbf{3}(\mathbf{4})$ & $<0.001$ \\
\hline Mean (SD) IPSS & $\mathbf{2 0}(\mathbf{8})$ & $\mathbf{9 ( 7 )}$ & $<0.001$ \\
\hline Mean (SD) IPSS QoL & $\mathbf{4 ( 1 )}$ & $\mathbf{2 ( 2 )}$ & $<0.001$ \\
\hline Mean (SD) BII & $\mathbf{7 ( 3 )}$ & $\mathbf{3 ( 3 )}$ & $<0.001$ \\
\hline $\begin{array}{l}\text { Mean (SD) MISI } \\
\text { Total }\end{array}$ & $\mathbf{7 ( 6 )}$ & $\mathbf{9 ( 8 )}$ & $\mathbf{0 . 0 0 7}$ \\
\hline $\begin{array}{l}\text { Mean (SD) MISI } \\
\text { Bother }\end{array}$ & $\mathbf{2 ( 2 )}$ & $2(3)$ & 0.588 \\
\hline Mean (SD) SHIM & $10(9)$ & $10(9)$ & 0.683 \\
\hline $\begin{array}{l}\text { Mean (SD) MSHQ- } \\
\text { EJD }\end{array}$ & $\mathbf{8 ( 6 )}$ & $\mathbf{6 ( 4 )}$ & $\mathbf{0 . 0 0 1}$ \\
\hline $\begin{array}{l}\text { Mean (SD) Maximal } \\
\text { Urine Flow Rate in } \\
\text { Milliliters/Second }\end{array}$ & $\mathbf{7 . 9 ( 4 . 6 )}$ & $\mathbf{1 7 . 1 ( 1 0 . 4 )}$ & $<0.001$ \\
\hline $\begin{array}{l}\text { Mean (SD) Average } \\
\text { Urine Flow Rate in } \\
\text { Milliliters/Second }\end{array}$ & $\mathbf{4 . 6 ( 8 . 5 )}$ & $\mathbf{5 0 . 6 ( 9 4 . 9 )}$ & \\
\hline $\begin{array}{l}\text { Mean (SD) Post-void } \\
\text { Residual in }\end{array}$ & $\mathbf{1 6 7 ( 2 1 9 )}$ & $10.0(18.8)$ & \\
\hline Milliliters & & & 0.104 \\
\hline
\end{tabular}


Table 2. Correlations between Preoperative GAD-7 Score and Patient Characteristics

\begin{tabular}{|c|c|c|}
\hline Patient Characteristic & Spearman's Correlation Coefficient & p-value \\
\hline Age (Years) & -0.053 & 0.534 \\
\hline BMI & 0.017 & 0.841 \\
\hline Preop IPSS Total & 0.167 & 0.051 \\
\hline Preop IPSS QoL & 0.012 & 0.892 \\
\hline Preop BII & 0.202 & 0.019 \\
\hline Preop MISI Total & 0.260 & 0.002 \\
\hline Preop MISI Bother & 0.345 & $<0.001$ \\
\hline Preop SHIM & -0.122 & 0.158 \\
\hline Preop MSHQ-EJD & -0.191 & 0.031 \\
\hline $\begin{array}{l}\text { Preop Maximal Urine Flow } \\
\text { Rate }\end{array}$ & -0.076 & 0.572 \\
\hline $\begin{array}{l}\text { Preop Average Urine Flow } \\
\text { Rate }\end{array}$ & 0.020 & 0.885 \\
\hline Preop Post Void Residual & -0.094 & 0.466 \\
\hline Enucleated Prostate Weight & -0.097 & 0.257 \\
\hline Postop GAD-7 & 0.571 & $<0.001$ \\
\hline Postop IPSS Total & 0.100 & 0.248 \\
\hline Postop IPSS QoL & -0.096 & 0.277 \\
\hline Postop BII & 0.057 & 0.514 \\
\hline
\end{tabular}




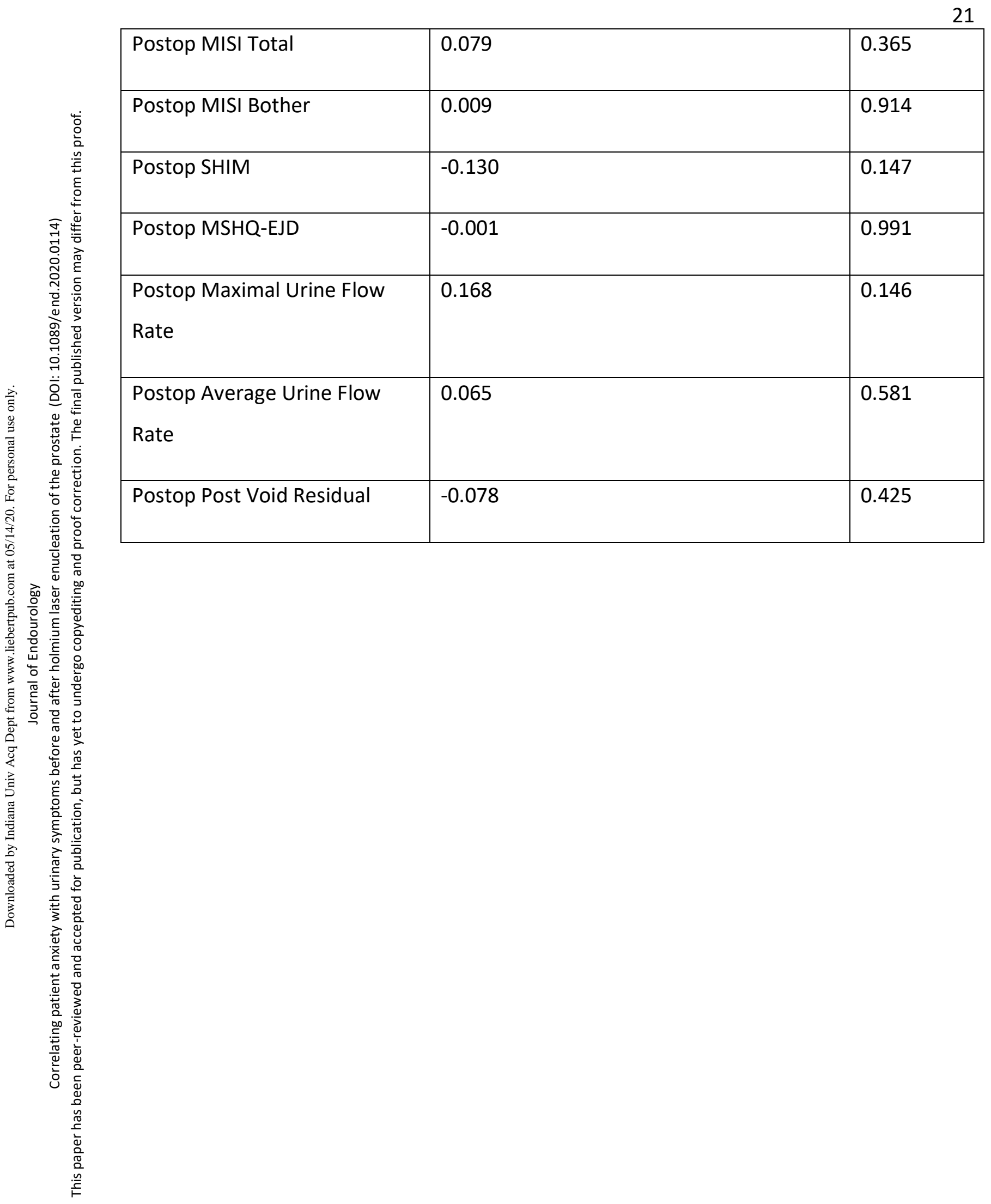


22

Supplementary Table 1. Comparison of preoperative and postoperative individual symptom scores within the IPSS.

\begin{tabular}{|l|l|l|l|}
\hline & Preoperative & Postoperative & p-value \\
\hline $\begin{array}{l}\text { Mean (SD) Incomplete Bladder } \\
\text { Emptying }\end{array}$ & $2.9(1.81)$ & $1.0(1.5)$ & $<0.001$ \\
\hline Mean (SD) Frequency & $3.5(1.5)$ & $2.0(1.7)$ & $<0.001$ \\
\hline Mean (SD) Intermittency & $2.9(1.7)$ & $0.8(1.3)$ & $<0.001$ \\
\hline Mean (SD) Urgency & $2.8(1.7)$ & $1.7(1.7)$ & $<0.001$ \\
\hline Mean (SD) Weak Stream & $3.3(1.7)$ & $0.7(1.3)$ & $<0.001$ \\
\hline Mean (SD) Nocturia & $2.7(1.4)$ & $1.8(1.3)$ & $<0.001$ \\
\hline
\end{tabular}


Supplemental Table 2. Sub-analysis of correlations between preoperative GAD-7 Score and individual symptom scores within the IPSS.

\begin{tabular}{|c|c|c|}
\hline Patient Characteristic & Spearman's Correlation Coefficient & p-value \\
\hline $\begin{array}{l}\text { Preop Incomplete Bladder } \\
\text { Emptying }\end{array}$ & 0.152 & 0.081 \\
\hline Preop Frequency & 0.131 & 0.134 \\
\hline Preop Intermittency & 0.030 & 0.733 \\
\hline Preop Urgency & 0.185 & 0.033 \\
\hline Preop Weak Stream & 0.077 & 0.376 \\
\hline Preop Straining & 0.173 & 0.048 \\
\hline Preop Nocturia & 0.158 & 0.071 \\
\hline $\begin{array}{l}\text { Postop Incomplete Bladder } \\
\text { Emptying }\end{array}$ & 0.086 & 0.397 \\
\hline Postop Frequency & 0.171 & 0.090 \\
\hline Postop Intermittency & 0.027 & 0.789 \\
\hline Postop Urgency & 0.201 & 0.045 \\
\hline Postop Weak Stream & -0.108 & 0.285 \\
\hline Postop Straining & 0.031 & 0.759 \\
\hline Postop Nocturia & 0.049 & 0.629 \\
\hline
\end{tabular}

